\title{
Three-dimensional analysis of craniofacial bones and soft tissues in obstructive sleep apnea using cone beam computed tomography
}

\section{Analyse tridimensionnelle des os craniofaciaux et des tissus mous dans l'apnée obstructive du sommeil utilisant la tomographie volumétrique à faisceau conique}

\author{
Annick BRUWIER ${ }^{\mathrm{a}, *}$, Robert POIRRIER $^{\mathrm{b}}$, Adelin ALBERT $^{\mathrm{c}}$, Nathalie MAES ${ }^{\mathrm{d}}$, \\ Michel LIMME ${ }^{\mathrm{a}}$, Carole CHARAVET ${ }^{\mathrm{a}}$, Mladen MILICEVIC ${ }^{\mathrm{e}}$, Sylvianne RASKIN ${ }^{\mathrm{a}}$, \\ Anne-Lise POIRRIER ${ }^{\mathrm{f}}$ \\ ${ }^{a}$ Department of Orthodontics, Liege University Hospital, Liege, Belgium \\ ${ }^{\mathrm{b}}$ Sleep Disorder Center, Department of Neurology, Liege University Hospital, Liege, Belgium \\ ${ }^{\mathrm{c}}$ Biostatistics, Liege University Hospital, Liege, Belgium \\ ${ }^{\mathrm{d}}$ Department of Biostatistics and Medico-Economic Information, Liege University Hospital, \\ Liege, Belgium \\ ${ }^{\mathrm{e}}$ Department of Medical Imaging, Liege University Hospital, Liege, Belgium \\ ${ }^{\mathrm{f}}$ ENT Department, Liege University Hospital, Liege, Belgium
}

Available online: 8 November 2016 / Disponible en ligne : 8 novembre 2016

\section{Summary}

A total of 154 adult patients with sleep complaints underwent a polysomnography and a craniofacial cone beam computed tomography (CBCT). OSA was defined as an apnea and hypopnea index $(A H I)$ or an oxygen desaturation index $(O D I) \geq 10$. Soft tissues and craniofacial bones volumes were prospectively measured by CBCT and collected blindly from sleep polysomnography. Among the study patients, 127 (83\%) suffered from OSA and 27 (17\%) did not. OSA patients demonstrated a narrower maxillo-palatine core volume (11.7 \pm 3.2 vs $14.6 \pm 4.9 \mathrm{~cm}^{3}$ ) even when adjusting for age, gender, height, neck circumference and body mass index. These upper airway measures provide a comprehensive analysis of bony structures and soft tissues, which can be involved in OSA.

\section{Résumé}

Un total de 154 patients souffrant de problèmes du sommeil ont subi une polysomnographie et une tomographie volumétrique à faisceau conique (cone beam ou CBCT). L'apnée obstructive du sommeil (AOS) a été définie comme une apnée avec un indice d'apnée ou d'hypoapnée $(I A H) \geq 10$. Les volumes des tissus mous et des os craniofaciaux ont été prospectivement mesurés par CBCT et enregistrés en aveugle par polysomnographie du sommeil. Parmi les patients dans l'étude, 127 (83\%) souffraient d'une AOS et 27 (17\%) n'en souffraient pas. Les patients AOS affichaient un volume total maxillopalatin plus étroit $\left(11,7 \pm 3,2\right.$ vs $\left.14,6 \pm 4,9 \mathrm{~cm}^{3}\right)$ même après ajustement en fonction de l'âge, du sexe, de la taille, de la circonférence du cou et de l'indice de masse corporelle. Ces valeurs des voies respiratoires supérieures

* Correspondence and reprints / Correspondance et tirés à part. e-mail address / Adresse e-mail : abruwier@yahoo.fr (Annick Bruwier) 
(C) 2016 CEO. Published by Elsevier Masson SAS. All rights reserved

\section{Key-words}

- Obstructive sleep apnea.

- Maxilla.

- Soft tissues.

- Cone beam.

\section{Introduction}

Obstructive sleep apnea (OSA) is a highly prevalent syndrome, amounting $9 \%$ in women and $24 \%$ in men. It is characterized by repetitive collapse of the upper airway (UA) during sleep [1]. The collapse alters gaseous exchanges and decreases the arterial blood saturation in oxygen below $90 \%$. The apnea and hypopnea index (AHI) is defined as the number of apnea and hypopnea per hour and the oxygen desaturation index (ODI) as the number of arterial blood desaturations in oxygen under $90 \%$ per hour. Today, the gold standard treatment for OSA is "continuous positive airway pressure" but nearly one third of the patients have difficulty using it. Alternative treatments include ENT, maxillofacial and bariatric surgery [2,3], stimulating device implantation [4] and oral appliance therapy [5]. A key aspect for alternative treatments consists in selecting the appropriate one in order to tackle specific anatomical issues of a given patient. Some OSA patients exhibit mandibular and maxillary deficiencies, caudally placed hyoid bone or shortening of the anterior skull base $[6,7]$.

Skeletal narrowness could be a poor outcome factor for soft tissue surgery. Orthodontists are increasingly concerned by this kind of OSA. They are closely implicated in the diagnosis and treatment of this syndrome within a multidisciplinary team composed essentially by physicians.

In recent years, extensive efforts have been spent in medical imaging to improve quality and reduce radiation exposure. By using a cone shaped X-ray beam, moving around the head in a circular motion, cone beam computed tomography (CBCT) has dramatically reduced radiation dosage, cost and time needed as compared to the conventional scanner. With its simplicity of use and high image quality, CBCT has become increasingly popular in orthodontic clinics [8]. Craniofacial measurements derived from CBCT are reproducible and valid [9,10]. CBCT has been used to evaluate maxillary canine impactions and labio-palatal clefts. It is believed that CBCT may help improving OSA assessment by providing a comprehensive and permettent d'élaborer une analyse très complète des structures osseuses et des tissus mous potentiellement impliqués dans l'AOS.

(C) 2016 CEO. Édité par Elsevier Masson SAS. Tous droits réservés

\section{Mots-clés}

- Apnée obstructive du sommeil.

- Maxillaire.

- Tissus mous.

- Cone beam.

\section{Introduction}

L'apnée obstructive du sommeil (AOS) est un syndrome très répandu touchant $9 \%$ de femmes et $24 \%$ d'hommes. Elle est caractérisée par des collapsus répétés des voies aériennes supérieures (VAS) pendant le sommeil [1]. Les collapsus altèrent les échanges gazeux et diminuent la saturation artérielle en oxygène à moins de $90 \%$. L'index d'apnée/hypopnée (IAH) est défini comme le nombre d'apnées et d'hypopnées par heure et l'index de désaturation en oxygène (IDO) comme le nombre de désaturations artérielles en oxygène à moins $90 \%$ par heure. De nos jours, le traitement de référence pour l'AOS est la « ventilation à pression positive continue » mais près d'un tiers des patients ont des difficultés à l'utiliser. Les traitements alternatifs comprennent l'ORL, la chirurgie maxillofaciale et bariatrique [2,3], la pose d'un stimulateur des VAS [4] et les traitements par pose d'un appareil oral [5]. Un élément essentiel des traitements alternatifs consiste à choisir l'option la plus appropriée pour aborder les problèmes anatomiques spécifiques chez un patient donné. Certains patients avec AOS montrent des déficits mandibulaires et maxillaires, un os hyoïde en position postérieure, ou un raccourcissement de la base crânienne antérieure $[6,7]$.

L'étroitesse squelettique peut constituer un facteur de risque d'un résultat médiocre en chirurgie des tissus mous. Les praticiens orthodontistes sont de plus en plus concernés par ce type d'AOS. Ils sont étroitement impliqués dans le diagnostic et le traitement de ce syndrome dans le cadre d'équipes pluridisciplinaires composées essentiellement de médecins.

Depuis quelques années, des efforts importants ont été réalisés en imagerie médicale pour améliorer la qualité des images et réduire l'exposition au rayonnement. L'utilisation d'un faisceau radiographique conique (CBCT ou cone beam en anglais), qui circule autour de la tête du patient, a permis de réduire de façon drastique les doses de radiation, le coût et le temps requis par ces images comparées à celles d'un scanner conventionnel. Grâce à sa facilité d'emploi et à la grande qualité de ses images, le cone beam est de plus en plus utilisé par les orthodontistes [8]. Les prises de mesure craniofaciales avec le cone beam sont valides et reproductibles $[9,10]$. Le cone beam a été utilisé pour évaluer les impactions de canines 
3-dimensional analysis of the upper airway while being fast and easy to interpret [11].

The aim of this study was to evaluate the diagnostic ability of CBCT in assessing craniofacial features (soft tissues and bones) of OSA patients in addition to demographic and anthropometric characteristics such as age, gender, height, neck circumference and body mass index (BMI).

\section{Material and methods}

The study was approved by the Ethics Committee of the University Hospital of Liege. A series of 154 consecutive Caucasoid adult patients, 57 women and 97 men consulting the Sleep Disorder Clinic of the University Hospital of Liege were recruited between April 2014 and May 2015. A full-night polysomnography was performed on each of them according to the American Academy of Sleep Medicine Manual for the Scoring of Sleep and Associated Events [12].

Subjects were diagnosed as OSA if their AHI was $\geq 10$ per hour or if their ODI was $\geq 10$ desaturations per hour of sleep; otherwise, they were considered as non-OSA.

Each subject also underwent a craniofacial CBCT performed with the New Tom 5G (New Tom, Verona), followed by a threedimensional analysis using the software Dolphin Imaging 11.7. Low CBCT resolution at $300 \mu \mathrm{m}$ decreased the X-ray exposure, while being adequate for the study purposes. During $\mathrm{X}$-raying, patients were in supine position, at the end of expiration and in maximum intercuspidation. They were asked to stop swallowing and breathing. The occlusal plane of the patient was perpendicular to the floor. The field of view was $16 \times 18 \mathrm{~cm}$. CBCT landmarks are detailed in $f i g .1$ and Table I.

Particular attention was paid to the craniofacial bones volumes. The maxillo-palatine core volume was defined from the two maxillae and palatine bones, made up of most maxillae's bodies and including the palatine processes and the horizontal plates of the palatine bones. The other maxilla processes (zygomatic, frontal and alveolar) were excluded (fig. 2). The maxillo-palatine core volume was defined anteriorly by the anterior border of the maxillae bodies, posteriorly by the posterior edges of the palatine processes, and laterally by the junctions with the zygomatic processes. As such, we defined a bone volume inside a parallelepiped, which enabled us to assess the roof of the mouth and the floor of the nasal cavities. Parameters also included volumes of the ethmoid and of the mandible, as well as areas of the sagittal median section of the tongue, the soft palate and the smallest transverse upper maxillaires et les fentes labiopalatines. Certains auteurs estiment que le cone beam pourrait améliorer l'évaluation des AOS en fournissant, de façon rapide et facile à interpréter, une analyse complète et tridimensionnelle des voies respiratoires supérieures [11].

L'objectif de cette étude était d'estimer les capacités diagnostiques du cone beam en évaluant les caractéristiques craniofaciales (tissus mous et os) de patients atteints d'AOS de même que leurs données démographiques et anthropométriques telles que leur âge, sexe, taille, circonférence du cou et indice de masse corporelle (IMC).

\section{Patients et méthodes}

L'étude a été approuvée par le Comité d'éthique du CHU de Liège. Une série de 154 patients adultes caucasiens consécutifs, 57 femmes et 97 hommes en consultation à la Clinique des troubles du sommeil du centre hospitalier universitaire de Liège ont été recrutés d'avril 2014 à mai 2015. Une polysomnographie se déroulant pendant une nuit entière de sommeil a été réalisée sur chaque patient en suivant le système d'évaluation du sommeil et des événements associés décrit dans le manuel de l'Académie américaine de la médecine du sommeil [12].

Le diagnostic d'AOS a été posé lorsque l'IAH des sujets était de $\geq 10$ par heure ou si leur IDO était de 10 désaturations par heure de sommeil. Sinon, ils étaient considérés comme nonAOS.

Chaque sujet a subi également un CBCT craniofacial en utilisant le New Tom 5G (New Tom, Verona) suivi d'une analyse tridimensionnelle à l'aide du logiciel Dolphin Imaging 11.7. La faible résolution du CBCT à $300 \mu \mathrm{m}$ a permis de réduire l'exposition au rayonnement de la radiographie tout en fournissant des images adéquates pour les besoins de l'étude. Pendant la radiographie, les patients étaient en position couchée, en fin d'expiration et en intercuspidie maximale. II leur a été demandé de ne pas déglutir ni de respirer. Le plan occlusal du patient était perpendiculaire au sol. Le champ de vision était de $16 \times 18 \mathrm{~cm}$. Les repères CBCT sont détaillés à la fig. 1 et au Tableau I.

Une attention particulière a été apportée aux volumes des os craniofaciaux. Le volume de base maxillopalatin a été défini à partir des deux maxillaires et des os palatins, constitués surtout des corps des maxillaires et comprenant les apophyses palatines et les parties horizontales des os palatins. Les autres apophyses maxillaires (zygomatiques, frontales et alvéolaires) ont été exclues (fig. 2). Le volume de base maxillopalatin a été défini antérieurement par la marge antérieure des corps maxillaires, postérieurement par les marges postérieures des apophyses palatines, et latéralement par les jonctions avec les apophyses zygomatiques. De cette façon, nous avons défini un volume osseux à l'intérieur d'un parallélépipède, ce qui nous a permis d'évaluer la voûte de la bouche et le plancher des cavités nasales. Les paramètres ont également inclus les volumes de l'ethmoïde et de la 

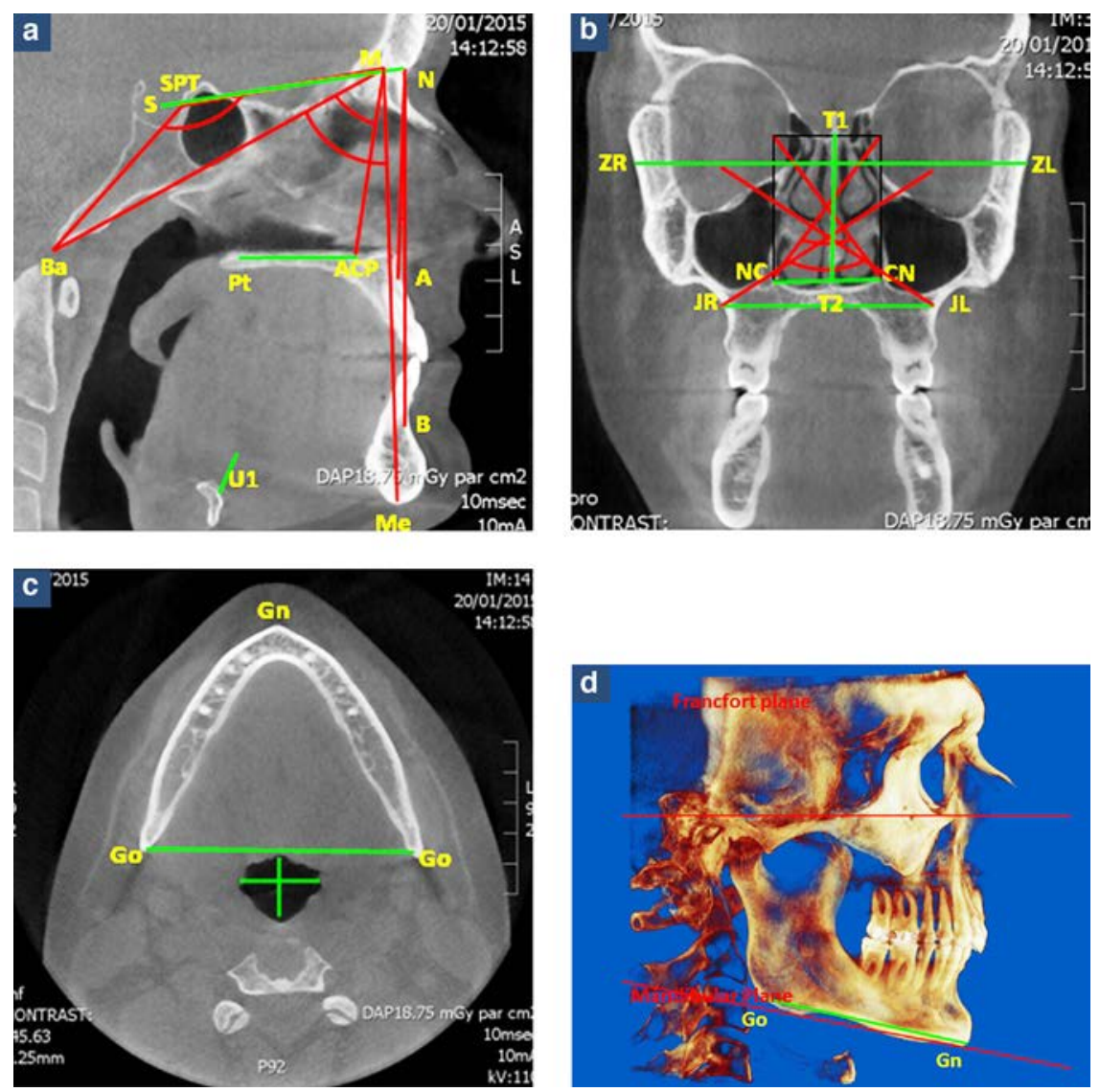

Fig. 1: a-d: angles and distances measured on CBCT images.

Fig. 1 : a-d : angles et distances mesurés sur des images CBCT.

airway section. The segmentation tool allowed us to isolate the mandible volume after removing the other craniofacial bones from the screen. The ethmoid bone segmentation was more complex than for the mandible: we had to identify each part of this bone, i.e. the Crista Galli, the cribriform plate, the perpendicular plate, the ethmoid sinus and the orbital plate of this ethmoid bone, which were intertwined with other bones. All CBCT parameters were collected independently and blindly from findings of the polysomnography.

Results were expressed as mean and standard deviation (SD) for quantitative variables and as frequencies and percentages for categorical variables. The association between height and craniofacial bones (ethmoid, maxilla and mandible) was measured by the Pearson correlation coefficient and analyzed by linear regression. OSA and non-OSA patients were compared by one-way analysis of variance or the Kruskal-Wallis test for skewed data distribution, while the chi-squared test was used mandibule aussi bien que des parties de la section médiane sagittale de la langue, le palais mou et la section transversale la plus petite des voies respiratoires supérieures. L'outil de segmentation nous a permis d'isoler le volume mandibulaire après avoir éliminé les autres os craniofaciaux de l'écran. La segmentation de l'os ethmoïde s'est avérée plus complexe que celle de la mandibule. Il a fallu identifier chaque partie de cet os, à savoir, la crista galli, la plaque cribriforme, la plaque perpendiculaire, le sinus ethmoïdal et la plaque orbitale de cet os ethmoïde, qui étaient imbriqués dans d'autres structures osseuses. Tous les paramètres CBCT ont été recueillis indépendamment et en aveugle à partir des résultats de la polysomnographie.

Les résultats ont été exprimés en tant que moyennes et écarts-types (ET) pour les variables quantitatives et comme fréquences et pourcentages pour les variables catégorielles. L'association entre la taille et les os craniofaciaux (ethmoïdes, maxillaires et mandibulaires) a été évaluée avec le coefficient de corrélation de Pearson et a été analysée par régression linéaire. Les patients avec ou sans AOS ont été comparés par analyse unidirectionnelle ou par le test de Kruskai-Wallis pour la distribution de données biaisées, alors que le test de $\mathrm{Chi}^{2}$ a 


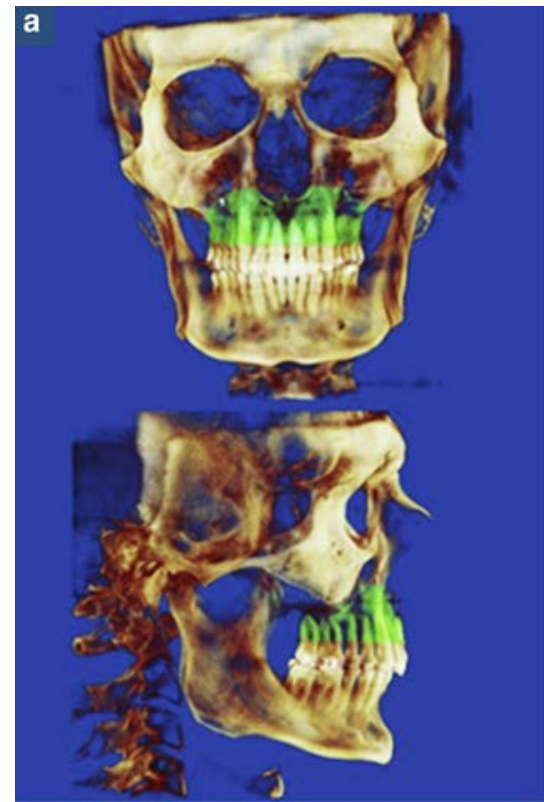

Fig. 2: Maxillo-palatine core volume. a: in a non-OSA patient $\left(14.9 \mathrm{~cm}^{3}\right)$. b: in an OSA subject $\left(10.8 \mathrm{~cm}^{3}\right)$. Fig. 2 : Volume de base maxillopalatin. a : chez un patient non-AOS $\left(14,9 \mathrm{~cm}^{3}\right)$. b : chez un sujet AOS $\left(10,8 \mathrm{~cm}^{3}\right)$.

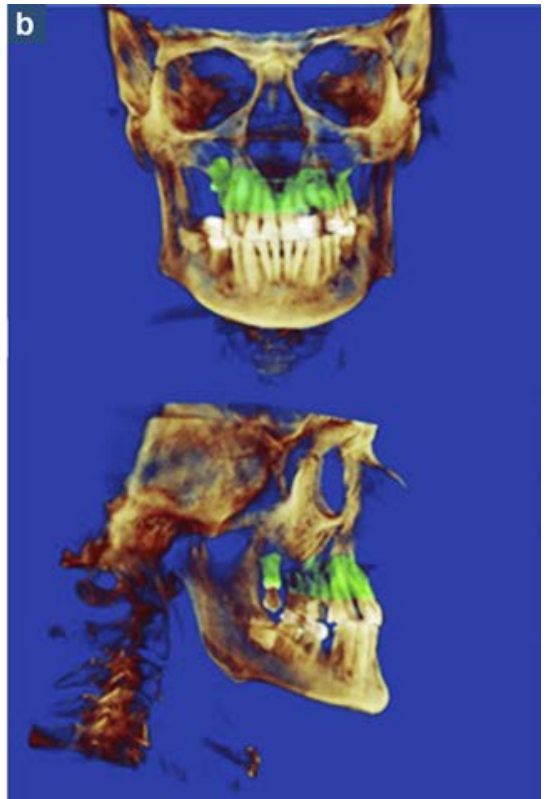

été utilisé pour comparer les proportions. Pour ajuster en fonction de l'âge, du sexe, de la taille, de la circonférence du cou et de l'IMC, une analyse de régression a été exécutée. Les résultats étaient significatifs à la valeur critique de $5 \%$ $(p<0,05)$. Les analyses ont été réalisées avec SAS (version 9.4 pour Windows) et $R$ (version 3.0.3).

\section{Résultats}

Les 154 sujets dans l'étude comprenaient 57 (37\%) femmes et 97 (63\%) hommes. Leur âge moyen était de $51 \pm 13$ ans (extrêmes : 22-78 ans. Leur taille moyenne était de $172 \pm 10 \mathrm{~cm}$ et leur IMC de $30,5 \pm 5,6 \mathrm{~kg} / \mathrm{m}^{2}$. Leurs caractéristiques démographiques, anthropométriques et celles relevées sur CBCT sont résumées au Tableau II (colonne 1). Dans l'ensemble, une corrélation significative forte a été relevée entre le volume de base maxillopalatin et le volume mandibulaire $r=0,64, p<0,0001)$. Les deux volumes étaient en relation positive avec la hauteur (pente $\pm \mathrm{SE}$ ) : le volume total maxillopalatin $(0,10 \pm 0,03, p=0,0008)$ et le volume mandibulaire $(0,39 \pm 0,058, p<0,0001)$. Par contre, aucune corrélation n'a été trouvée entre la hauteur et le volume de l'os ethmoïde $(p=0,61)$ (fig. 3).

L'examen polysomnographique a fourni un IAH moyen de $33,8 \pm 26,7$ et un IDO moyen de $29,1 \pm 25,5$. Comme le 


\section{Table I}

Angles (degrees), distances $(\mathrm{cm})$, volumes $\left(\mathrm{cm}^{3}\right)$ and surfaces $\left(\mathrm{cm}^{2}\right)$ measured on CBCT images.

\section{Tableau I}

Angles (degrés), distances $(\mathrm{cm})$, volumes $\left(\mathrm{cm}^{3}\right)$ et surfaces $\left(\mathrm{cm}^{2}\right)$ mesurés sur les images CBCT.

\begin{tabular}{|c|c|c|}
\hline \multirow[t]{7}{*}{ Angle } & ANB angle & $\begin{array}{l}\text { Maxillo-mandibular sagittal discrepancy/Décalage sagittal } \\
\text { maxillomandibulaire }\end{array}$ \\
\hline & Ba-SPT-M & $\begin{array}{l}\text { Basion-superior pituitary tubercle-frontonasomaxillary suture/Basion - } \\
\text { tubercule pituitaire supérieur - frontonasomaxillaire }\end{array}$ \\
\hline & FMA & $\begin{array}{l}\text { Facial mandibular divergence (Francfort Plane-Mandibular Plane)/ } \\
\text { Divergence faciale mandibulaire (Plan Francfort-Plan mandibullaire) }\end{array}$ \\
\hline & Ba-M-Me & $\begin{array}{l}\text { Basion-M (intersection of nasal, frontal and maxillary bones)-chin angle/ } \\
\text { Basion-M (intersection des os nasaux, frontaux, et maxillaires bones)-angle } \\
\text { menton }\end{array}$ \\
\hline & Ba-M-APC & $\begin{array}{l}\text { Basion-M-anterior palatal canal angle/Angle Basion-M antérieur conduit } \\
\text { palatin }\end{array}$ \\
\hline & $\begin{array}{l}\text { Nasal diagonals/ } \\
\text { Nasal diagonals }\end{array}$ & $\begin{array}{l}\text { Angle between the diagonals drawn inside the nasal fossae coronal section/ } \\
\text { Angle entre les diagonales tracées à l'intérieur de la section coronale de la } \\
\text { cavité nasale section }\end{array}$ \\
\hline & $\begin{array}{l}\text { Maxillary diagonals/ } \\
\text { Maxillaire diagonals }\end{array}$ & $\begin{array}{l}\text { Angle between the diagonals drawn inside the maxillary coronal section/ } \\
\text { Angle entre les diagonales tracées à l'intérieur de la section corcoronales } \\
\text { coronale ronal section }\end{array}$ \\
\hline \multirow[t]{11}{*}{ Distance } & SN (anterior skull base) & $\begin{array}{l}\text { Distance between the sphenoidal pituitary tubercle and the nasion/Distance } \\
\text { entre la turbercule hypophysaire sphénoidale et tubercle and the nasion }\end{array}$ \\
\hline & JR-JL & $\begin{array}{l}\text { Maxillary width (between the concavity of the maxillary tuberosities)/Largeur } \\
\text { maxillaire (entre la concavité des tubérosités maxillaires lary tuberosities) }\end{array}$ \\
\hline & $\overline{\mathrm{Pt}-\mathrm{APC}}$ & $\begin{array}{l}\text { Maxillary length (floor of the nasal fossae)/Longueur maxillaire (plancher de la } \\
\text { cavité nasale) }\end{array}$ \\
\hline & $\overline{\mathrm{ZR}-\mathrm{ZL}}$ & Biorbital width/Largeur bi-orbitaire \\
\hline & NC-CN & Nasal width/Largeur nasale \\
\hline & $\mathrm{T} 1-\mathrm{T} 2$ & Nasal height/Hauteur nasale \\
\hline & Go-Go & Mandibular width/Largeur mandibulaire \\
\hline & Gn-Go & Mandibular length/Longueur mandibulaire \\
\hline & U1-MP & Hyoid bone-mandibular plane/Plan os hyoïdien-plan mandibulaire \\
\hline & UA width/Largeur VAS & Smallest UA section width/Largeur de la plus petite section VAS \\
\hline & UA length/Longueur VAS & Smallest UA section length/Longueur de la plus petite section VAS \\
\hline \multirow{3}{*}{$\begin{array}{l}\text { Volume areas/ } \\
\text { Volume }\end{array}$} & Ethmoid/Ethmoïdal & Volume of the ethmoid/Volume de l'ethmoïde \\
\hline & $\begin{array}{l}\text { Maxillo-palatine core/ } \\
\text { Maxillopalatin core }\end{array}$ & $\begin{array}{l}\text { Bodies of both maxillae and palatine bones/Corps des deux maxillaires et des } \\
\text { os palatins }\end{array}$ \\
\hline & Mandible/Mandibulaire & Volume of the mandible/Volume de la mandibule \\
\hline \multirow[t]{3}{*}{ Surface } & UA surface/Surface VAS & $\begin{array}{l}\text { Smallest transverse upper airway section/Plus petite section transversale des } \\
\text { voies aériennes supérieures aériennes }\end{array}$ \\
\hline & Tongue surface/Surface langue & Sagittal median section of the tongue/Section médiane sagittale de la langue \\
\hline & $\begin{array}{l}\text { Soft palate surface/Surface } \\
\text { Palais mou }\end{array}$ & $\begin{array}{l}\text { Sagittal median section of the soft palate/Section médiane sagittale du palais } \\
\text { mou }\end{array}$ \\
\hline
\end{tabular}


Three-dimensional analysis of craniofacial bones and soft tissues in obstructive sleep apnea using cone beam computed tomography Analyse tridimensionnelle des os craniofaciaux et des tissus mous dans l'apnée obstructive du sommeil utilisant la tomographie

volumétrique à faisceau conique

\section{Table II}

Overall characteristics of study patients $(n=154)$ and comparison of OSA and non-OSA patients.

\section{Tableau II}

Caractéristiques globales des patients dans l'étude $(n=154)$ et comparaison des patients AOS et non-AOS.

\begin{tabular}{|c|c|c|c|c|}
\hline Characteristics/Caractéristiques & $\begin{array}{l}\text { Global } \\
n=154\end{array}$ & $\begin{array}{l}\text { Non-OSA/ } \\
\text { Non AOS } \\
n=27\end{array}$ & $\begin{array}{l}\text { OSA/AOS } \\
n=127\end{array}$ & $\begin{array}{l}P \text {-value/ } \\
\text { Valeur } p\end{array}$ \\
\hline Age (years)/Áge (ans) & $52 \pm 13$ & $45 \pm 17$ & $53 \pm 11$ & 0.0032 \\
\hline Gender (male)/Sexe (homme) & $97(63 \%)$ & $12(44 \%)$ & $85(67 \%)$ & 0.031 \\
\hline$\overline{\mathrm{AHI}}$ (events/h of sleep)/IAH (épisodes/h de sommeil) & $33.8 \pm 26.7$ & $4.6 \pm 3.4$ & $40.0 \pm 25.3$ & $<0.0001$ \\
\hline ODI (events/h of sleep)/IDO (épisodes/h de sommeil) & $29.1 \pm 25.5$ & $2.9 \pm 2.7$ & $34.7 \pm 24.7$ & $<0.0001$ \\
\hline Neck circumference $(\mathrm{cm}) /$ Circonférence du cou $(\mathrm{cm})$ & $42.0 \pm 5.0$ & $38.2 \pm 3.4$ & $43.0 \pm 4.9$ & $<0.0001$ \\
\hline Height (cm)/Taille (cm) & $172 \pm 10$ & $171.9 \pm 9.4$ & $171.5 \pm 10.1$ & 0.88 \\
\hline BMI $\left(\mathrm{kg} / \mathrm{m}^{2}\right) / I M C\left(\mathrm{~kg} / \mathrm{m}^{2}\right)$ & $30.5 \pm 5.6$ & $27.1 \pm 5.7$ & $31.3 \pm 5.3$ & 0.0007 \\
\hline Epworth score (/24)/Score Epworth (/24) & $10.8 \pm 5.1$ & $12.9 \pm 5.0$ & $10.4 \pm 5.0$ & 0.029 \\
\hline \multicolumn{5}{|l|}{ Angles () } \\
\hline ANB & $3.5 \pm 2.9$ & $3.4 \pm 3.4$ & $3.5 \pm 2.9$ & 0.83 \\
\hline Ba-SPT-M & $133.3 \pm 5.9$ & $132.9 \pm 5.2$ & $133.4 \pm 6.0$ & 0.71 \\
\hline FMA & $23.1 \pm 5.7$ & $23.1 \pm 3.8$ & $23.1 \pm 6.1$ & 0.99 \\
\hline Ba-M-Me & $59.3 \pm 4.5$ & $59.7 \pm 3.8$ & $59.2 \pm 4.7$ & 0.60 \\
\hline Ba-M-APC & $57.5 \pm 3.9$ & $57.6 \pm 4.3$ & $57.5 \pm 3.8$ & 0.92 \\
\hline $\begin{array}{l}\text { Angle between nasal diagonals/Angle entre les } \\
\text { diagonales nasales }\end{array}$ & $72 \pm 6.1$ & $74.4 \pm 6.3$ & $71.5 \pm 6.0$ & 0.028 \\
\hline $\begin{array}{l}\text { Angle between maxillary diagonals/ } \\
\text { Angle entre les diagonales maxillaires }\end{array}$ & $102.6 \pm 7.3$ & $105.1 \pm 7.2$ & $102.0 \pm 7.3$ & 0.051 \\
\hline \multicolumn{5}{|l|}{ Distances } \\
\hline SN distance $(\mathrm{cm}) /$ Distance $S N(\mathrm{~cm})$ & $6.8 \pm 0.37$ & $6.9 \pm 0.40$ & $6.8 \pm 0.36$ & 0.71 \\
\hline $\begin{array}{l}\text { Maxillary width JR-JL }(\mathrm{cm}) / \text { Largeur } \\
\text { maxillaire } J R-J L(\mathrm{~cm})\end{array}$ & $5.6 \pm 0.58$ & $5.7 \pm 0.67$ & $5.6 \pm 0.56$ & 0.80 \\
\hline $\begin{array}{l}\text { Maxillary length Pt-APC }(\mathrm{cm}) / \text { Longueur } \\
\text { maxillaire Pt-APC }(\mathrm{cm})\end{array}$ & $3.8 \pm 0.32$ & $3.8 \pm 0.30$ & $3.8 \pm 0.33$ & 0.86 \\
\hline $\begin{array}{l}\text { Biorbital width ZR-ZL }(\mathrm{cm}) / \text { Largeur } \\
\text { bi-orbitaire ZR-ZL (cm) }\end{array}$ & $10.5 \pm 0.53$ & $10.5 \pm 0.55$ & $10.5 \pm 0.53$ & 0.74 \\
\hline $\begin{array}{l}\text { Nasal width NC-CN }(\mathrm{cm}) / \text { Largeur } \\
\text { nasale NC-CN }(\mathrm{cm})\end{array}$ & $3.1 \pm 0.36$ & $3.2 \pm 0.43$ & $3.1 \pm 0.35$ & 0.30 \\
\hline Nasal height $(\mathrm{cm}) /$ Hauteur nasale $(\mathrm{cm})$ & $4.7 \pm 3.2$ & $4.4 \pm 0.48$ & $4.8 \pm 3.6$ & 0.24 \\
\hline Mandibular width $(\mathrm{cm}) /$ Largeur mandibulaire $(\mathrm{cm})$ & $9.7 \pm 0.7$ & $9.6 \pm 0.77$ & $9.7 \pm 0.68$ & 0.45 \\
\hline $\begin{array}{l}\text { Mandibular corpus length Go-Gn }(\mathrm{cm}) / \text { Longueur } \\
\text { du corps mandibulaire Go-Gn }(\mathrm{cm})\end{array}$ & $7 \pm 0.69$ & $7.1 \pm 0.84$ & $7 \pm 0.66$ & 0.97 \\
\hline $\begin{array}{l}\text { Hyoid bone/mandibular plane distance }(\mathrm{cm}) / \\
\text { Distance os hyoïdien/plan mandibulaire }(\mathrm{cm})\end{array}$ & $1.7 \pm 0.84$ & $1.2 \pm 0.77$ & $1.8 \pm 0.83$ & 0.0057 \\
\hline \multicolumn{5}{|l|}{$\begin{array}{l}\text { Smallest transverse UA section dimensions/ } \\
\text { Dimensions section transver/le la plus petite }\end{array}$} \\
\hline Sagittal $(\mathrm{mm}) /$ Sagittal $(\mathrm{mm})$ & $5.8 \pm 2.4$ & $6.6 \pm 2.5$ & $5.7 \pm 2.4$ & 0.064 \\
\hline Transverse (mm)/Transversal $(\mathrm{mm})$ & $14.7 \pm 6.1$ & $17.6 \pm 5.2$ & $14.2 \pm 6.2$ & 0.010 \\
\hline \multicolumn{5}{|l|}{ Volumes $\left(\mathrm{cm}^{3}\right)$} \\
\hline Ethmoid/Ethmoïde & $2.7 \pm 1.0$ & $3.3 \pm 1.6$ & $2.6 \pm 0.79$ & 0.0032 \\
\hline Maxillo-palatine core/Total maxillopalatin & $12.2 \pm 3.7$ & $14.6 \pm 4.9$ & $11.7 \pm 3.2$ & 0.0009 \\
\hline
\end{tabular}




\section{Table II}

Overall characteristics of study patients $(n=154)$ and comparison of OSA and non-OSA patients. (following)
Tableau II

Caractéristiques globales des patients dans l'étude $(n=154)$ et comparaison des patients AOS et non-AOS. (suite)

\begin{tabular}{llllc}
\hline Characteristics/Caractéristiques & $\begin{array}{l}\text { Global } \\
n=154\end{array}$ & $\begin{array}{l}\text { Non-OSA/ } \\
\text { Non AOS } \\
n=27\end{array}$ & $\begin{array}{l}\text { OSA/AOS } \\
n=127\end{array}$ & $\begin{array}{l}P \text {-value/ } \\
\text { Valeur } p\end{array}$ \\
\hline$\quad$ Mandible/Mandibule & $39.0 \pm 7.9$ & $39.2 \pm 8.7$ & $39.0 \pm 7.8$ & 0.88 \\
\hline $\begin{array}{l}\text { Surfaces }\left(\mathrm{cm}^{2}\right) \\
\begin{array}{l}\text { Sagittal section of the tongue/ } \\
\text { Section sagittale de la langue }\end{array}\end{array}$ & $27.5 \pm 4.3$ & $25.7 \pm 4.5$ & $27.9 \pm 4.2$ & 0.023 \\
\hline $\begin{array}{l}\text { Sagittal section of the soft palate/ } \\
\text { Section sagittale du palais mou }\end{array}$ & $3.1 \pm 0.89$ & $2.6 \pm 0.74$ & $3.3 \pm 0.88$ & 0.0007 \\
\hline $\begin{array}{l}\text { Smallest transverse UA section/ } \\
\text { Section transversale la plus petite des VAS }\end{array}$ & $0.84 \pm 0.49$ & $1.1 \pm 0.56$ & $0.79 \pm 0.46$ & 0.0087 \\
\hline
\end{tabular}
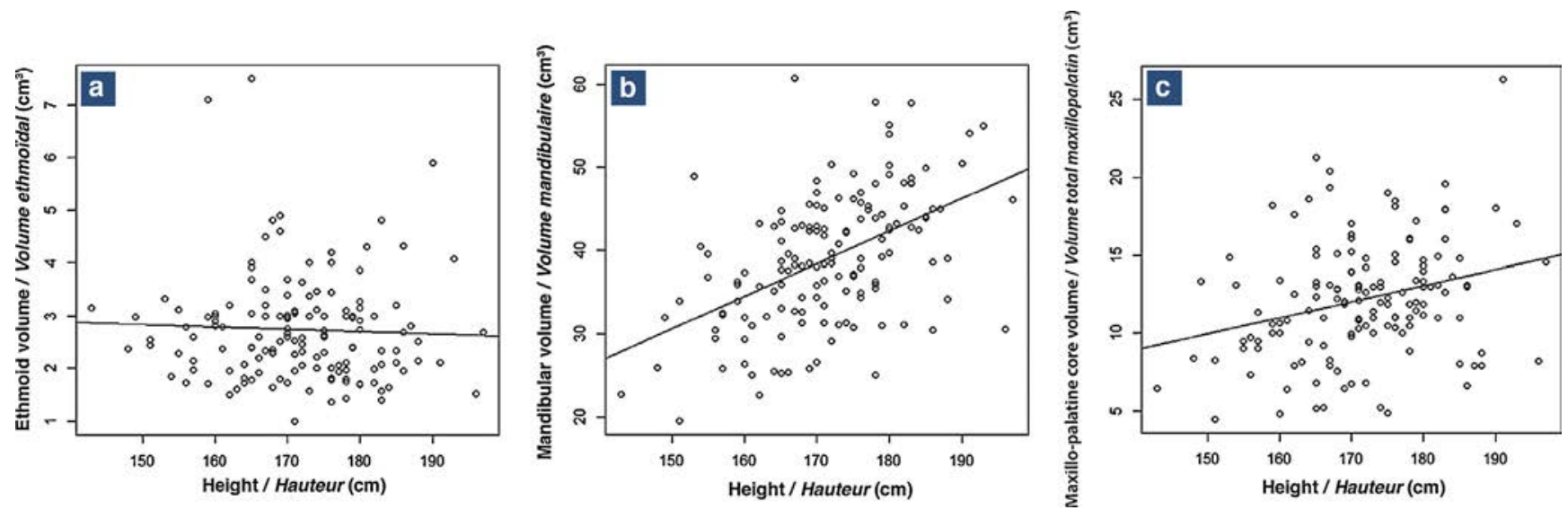

Fig. 3: Correlations between height (X).

a: maxillo-palatine core volume (graph A: $\mathrm{Y}=-5.5+0.10 \mathrm{X})$.

b: mandibular volume (graph B: $\mathrm{Y}=-28+0.39 \mathrm{X})$.

c: ethmoid volume (graph $\mathrm{C}$ : $\mathrm{Y}=3.5-0.0043 \mathrm{X}$ ).

Fig. 3 : Corrélations entre la taille $(\mathrm{X})$.

a : le volume de base maxillopalatin (graphique $A: Y=-5,5+0,10 X)$.

b : le volume mandibulaire (graphique $B: Y=-28+0,39 X)$.

c : le volume ethmoïdal (graphique $C: Y=3,5-0,0043 X$ ).

there was a strong correlation between the two indexes $(r=0.89 ; P<0.0001)$.

According to the OSA definition (AHI $\geq 10$ or ODI $\geq 10$ ), $127(83 \%)$ patients were diagnosed as having OSA and 27 (17\%) subjects were declared free of the syndrome. These proportions were representative of the population of subjects undergoing polysomnography in a tertiary referral hospital.

The comparison of OSA and non-OSA patients is given in Table II. The two groups differed significantly with respect to age, gender, neck circumference and BMI but were comparable with respect to height. OSA subjects were older, had a montre la fig. 4 , il existait une corrélation forte entre les deux index $(r=0,89 ; p<0,0001)$.

Selon la définition de l'AOS (IAH $\geq 10$ ou IDO $\geq 10$ ), 127 (83\%) des patients étaient diagnostiqués comme souffrant d'AOS et 27 (17\%) des sujets ont été déclarés exempts du syndrome. Ces proportions étaient représentatives de la population de sujets subissant une polysomnographie dans un hôpital de référence tertiaire.

La comparaison des patients avec ou sans AOS est donnée au Tableau II. Les deux groupes ont affiché des différences significatives en ce qui concerne l'âge, le sexe, la circonférence du cou et l'IMC mais étaient comparables en ce qui 


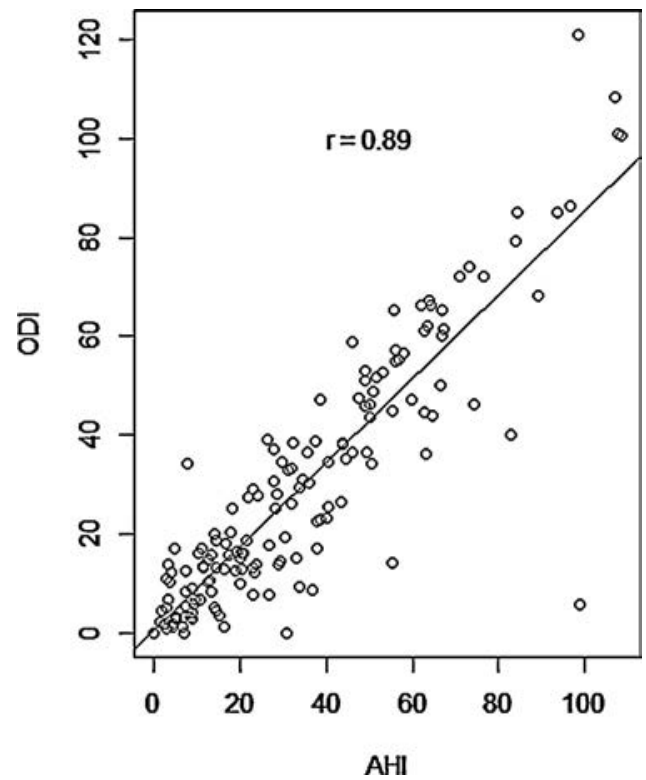

Fig. 4: Scatter plot of study patients $(n=154)$ according to AHI and ODI (regression line: $\mathrm{Y}=0.32$ 0.85.X).

Fig. 4 : Diagramme de dispersion des patients dans l'étude $(n=154)$ selon l'IAH et l'IDO (ligne de régression : $Y=0,320,85 X)$.

greater neck circumference and body mass index than the others. The proportion of males was also significantly higher in the OSA group than in the non-OSA group (67\% vs $44 \%)$.

Regarding the CBCT parameters, it is noteworthy that the sagittal sectional tongue and soft palate areas, as well as the hyoid bone/mandibular plane distance, were significantly larger in the OSA group. By contrast, the smallest transverse UA section, its transverse dimension and the angle between nasal diagonals were smaller in that group. The ethmoid and maxillo-palatine core volumes were also smaller in OSA patients. However, when adjusting for age, gender, height and BMI, logistic regression analysis showed that the maxillo-palatine core volume was the only bony structure which remained significantly smaller $(P=0.012)$ in the OSA group (fig. 2).

\section{Discussion}

All study subjects were admitted to the Sleep Disorder Clinic with subjective complaints of sleep disorders. The actual concerne la taille. Les sujets avec AOS étaient plus âgés, avaient une circonférence du cou plus grande et un indice de masse corporelle plus important que les non-AOS. La proportion d'hommes était également significativement plus élevée dans le groupe AOS par rapport au groupe non-AOS (67\% vs $44 \%$ ).

En ce qui concerne les paramètres relevés par $\mathrm{CBCT}$, il est à noter que la coupe sagittale de la langue et les régions du palais mou, de même que la distance os hyoïde/plan mandibulaire, étaient significativement plus importantes dans le groupe AOS. En contrepartie, la section transversale la plus petite des VAS, leur dimension transversale et l'angle entre les diagonales nasales étaient plus petits dans ce groupe. De même, les volumes de base ethmoïde et maxillopalatin étaient moins importants chez les patients AOS. Cependant, après ajustement en fonction de l'âge, du sexe, de la taille et de I'IMC, l'analyse de régression logistique a révélé que le volume de base maxillopalatin était la seule structure osseuse qui restait significativement plus petite $(p=0,012)$ dans le groupe AOS (fig. 2).

\section{Discussion}

Tous les sujets dans l'étude ont été admis à la Clinique des troubles du Sommeil pour des problèmes liés au sommeil. La 
presence or absence of sleep disorders was objectified by a full-night polysomnography examination and not just based on a subjective self-evaluated sleep questionnaire. It turned out that $83 \%$ were diagnosed as OSA-based on AHI and ODI values.

To the best of our knowledge, this is the first study measuring craniofacial bone volumes by CBCT in addition to classical polysomnographic parameters in patients with suspected OSA disorder. Given the large number of components of the maxillary base, which are subject to variations and do not show the bone volume around the maxillary airways, a manual selection of bone landmarks was operated to tentatively define the maxillo-palatine core volume; this may include an element of subjectivity. Indeed, the determination of this particular volume can be detrimental for the evaluation of the actual maxillary bone volume. To date, there is no accepted definition of the maxillo-palatine core volume and this aspect will have to be further explored. As for the mandible, the bone volume was easier to determine because the limits are clearly more visible. The determination of the ethmoid bone volume was a little bit more difficult than for the mandible because of the connections of this bone with the others. Whilst it would be interesting to know the nasal fossae volume, it is impossible for the time being, as the Dolphin Imaging 11.7 software does not have the capability to isolate the nasal fossae volume, as it is in contact with external air.

Since patients were examined and measured in supine position as in the sleep position, the study findings regarding positional variables should be considered with caution in comparison with traditional $2 \mathrm{D}$ and $3 \mathrm{D}$ cephalometry performed in upright or sitting positions [13]. This work based on CBCT shows how anatomical parameters differ in OSA and non-OSA patients, whether considering the soft tissues or the bony structures. CBCT is particularly efficient for measuring bone volumes and this study has clearly evidenced that OSA patients do have a narrower maxillo-palatine core volume.

As a reminder, both maxilla and mandible volumes were found correlated with height while the ethmoid bone was not. This characteristic observation is particularly relevant when it comes to interpreting CBCT measurements. Mandibular development is a consequence of reciprocal epigenetic interactions of both skeletal and non-skeletal compartments. The endochondral bony origin of the ethmoid labyrinths makes them substantially different and predominantly determined by genetics rather than by postnatal pressures [14]. Ethmoid growth stops by the age of 2 years. It is known that maxillary narrowness is a factor of OSA in children [15,16], which may be treated by orthodontic maxillary expansion combined or not with adenotonsillectomy [17]. The present study explicitly highlighted maxillo-palatine narrowness after height présence effective ou l'absence de troubles du sommeil a été objectivé par un examen polysomnographique durant toute une nuit et n'était pas basée uniquement sur un questionnaire subjectif autoévalué portant sur le sommeil. Quatre-vingt-trois pour cent des sujets ont été effectivement diagnostiqués avec AOS sur la base de valeurs d'IAH et d'IDO.

À notre connaissance, la présente étude est la première à évaluer les volumes des os craniofaciaux par CBCT associé à des paramètres polysomnographiques classiques chez des patients soupçonnés d'être atteints d'un trouble lié à l'AOS. Étant donné le nombre important de composants de la base maxillaire sujets à variations et ne participant pas au volume osseux entourant les voies respiratoires maxillaires, nous avons dressé une sélection manuelle montrant les repères osseux afin d'essayer de définir le volume de base maxillopalatin. Cette entreprise peut également inclure une part de subjectivité. En effet, la détermination de ce volume peut porter préjudice à l'évaluation du volume d'os maxillaire effectivement présent. Jusqu'à ce jour, il n'existe pas de définition claire du volume de base maxillopalatin et de nouvelles recherches sont nécessaires. Quant à la mandibule, le volume osseux de base a été plus facile à calculer puisque les limites sont plus nettement visibles. L'évaluation du volume de base de l'os ethmoïde s'est révélée plus difficile que pour la mandibule étant donné les liaisons de cet os avec les autres os. II serait intéressant, mais impossible pour l'instant, de connaître le volume de la fosse nasale en raison de l'incapacité du logiciel Dolphin Imaging 11.7 à isoler le volume de cette cavité puisque celle-ci est en contact avec l'air libre.

Du fait que les patients aient été examinés et mesurés en position allongée, comme pendant le sommeil, les résultats de cette étude en ce qui concerne les variables de position doivent être abordés avec précaution par rapport à la céphalométrie $2 \mathrm{D}$ et $3 \mathrm{D}$ traditionnelle réalisée en position debout ou assise [13]. Ces recherches basées sur l'imagerie $\mathrm{CBCT}$ révèlent les différences entre les paramètres anatomiques de patients avec et sans AOS, que l'on tienne compte des tissus mous ou des structures osseuses. Le cone beam est particulièrement efficace pour mesurer les volumes osseux et cette étude a clairement démontré que les patients souffrant d'AOS possèdent effectivement un volume de base maxillopalatin plus étroit.

Pour rappel, nous avons trouvé une corrélation entre les volumes des maxillaires et des mandibules et la taille mais aucune corrélation entre os ethmoïde et taille. Cette observation est particulièrement pertinente quand il s'agit d'interpréter les mesures CBCT. Le développement mandibulaire est la conséquence d'interactions épigénétiques réciproques entre les deux compartiments, squelettique et non squelettique. L'origine osseuse endochondrale des labyrinthes ethmoïdaux les rend significativement différents du fait qu'ils sont déterminés par la génétique beaucoup plus que par les pressions postnatales [14]. La croissance ethmoïdale s'arrête avant l'âge de 2 ans. II est bien connu que l'étroitesse maxillaire est un facteur d'AOS chez les enfants [15,16], qui peut être traitée par l'expansion maxillaire orthodontique associée, 
adjustment in adults suffering from OSA, as was previously suggested in cephalometric studies. This maxillo-palatine narrowness could be a residual consequence of oral breathing adopted much earlier in childhood. Mouth breathing in children alters the biophysical environment of the developing musculoskeletal system and can lead to a growth defect of the maxilla [18]. The maxilla is a major component of the nasal bony framework but it is not addressed with conventional septoplasty. This may explain the increase of nasal resistance in OSA, but also the failure of conventional nose surgery to treat OSA [19].

CBCT evaluation of soft tissue parameters associated with OSA was consistent with the findings of computed tomography [20] and magnetic resonance imaging studies [21]. Soft tissues thickness may be a cause (obesity, anatomical narrowness) or a consequence (swelling due to snoring) of OSA. CBCT may enhance clinical evaluation of the soft tissues, adding quantifiable parameters to dynamic examination.

This study has clearly revealed two kinds of anatomical factors for OSA: the maxillo-mandibular framework and soft tissues (fig. 5). The two little horizontal bars on fig. 5 show the smallest transverse UA section. For the patient on the left, a ou pas, à l'adénotonsillectomie [17]. L'étude présente a souligné explicitement l'étroitesse maxillopalatine après ajustement pour la taille chez les adultes souffrant d'AOS, comme cela a déjà été suggéré dans des études céphalométriques antérieures. Cette constriction maxillopalatine pourrait être la conséquence résiduelle de la respiration buccale adoptée bien antérieurement pendant l'enfance. La respiration buccale chez les enfants modifie l'environnement biophysique du système musculosquelettique en voie de croissance et peut aboutir à un défaut de croissance maxillaire [18]. Le maxillaire est une composante importante de l'ossature nasale mais qui n'est pas traité par la septoplastie conventionnelle, ce qui peut expliquer l'augmentation de la résistance nasale dans l'AOS, mais également l'incapacité de la rhinochirurgie traditionnelle à corriger l'AOS [19].

L'évaluation par CBCT des paramètres des tissus mous associés à l'AOS était en accord avec les résultats des images tomographiques [20] et les études basées sur l'imagerie à résonance magnétique [21]. L'épaisseur des tissus mous peut être soit une cause (obésité, étroitesse anatomique) soit une conséquence (enflure due au ronflement) de l'AOS. Le CBCT peut améliorer l'évaluation clinique des tissus mous en ajoutant des paramètres quantifiables à l'examen dynamique. Cette étude a clairement démontré deux sortes de facteurs anatomiques à l'origine de l'AOS : l'ossature maxillomandibulaire et les tissus mous (fig. 5). Les deux petites barres affichées à la fig. 5 montrent la section transversale la plus
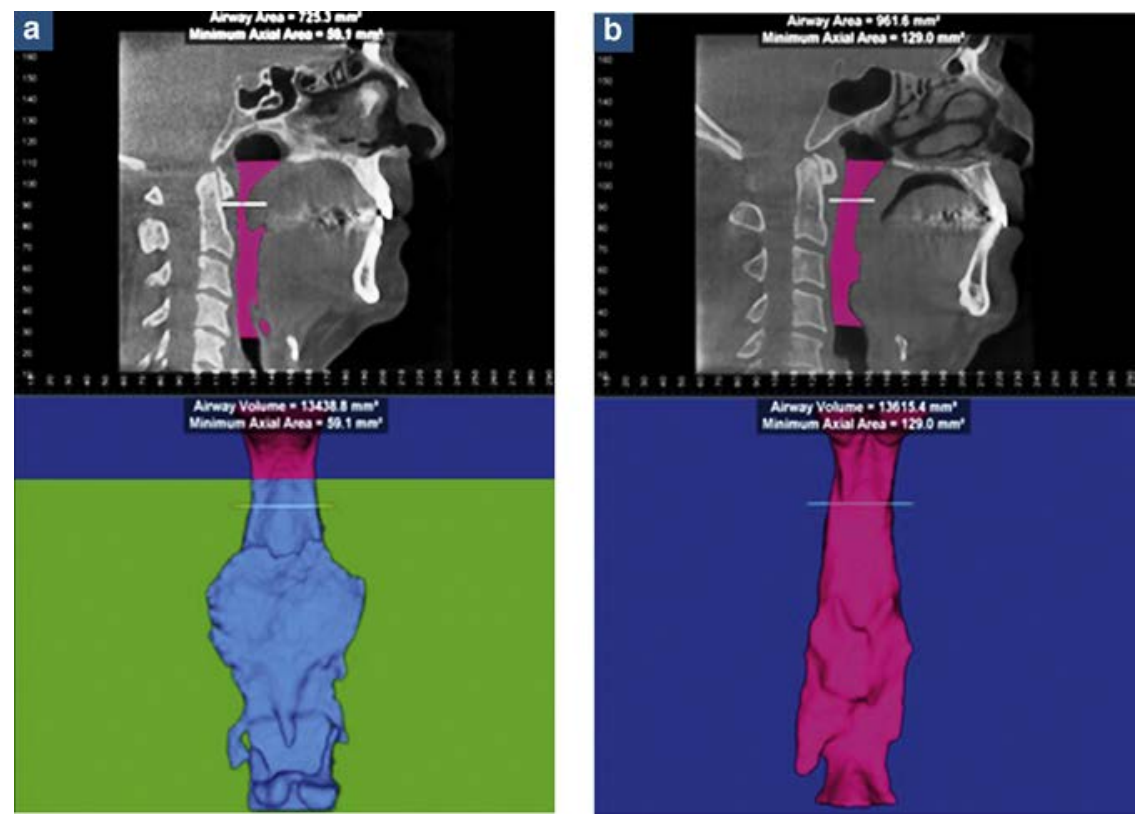

Fig. 5: Upper airway evaluation of two patients.

a: airway narrowness mainly due to maxillo-mandibular framework.

b: soft tissues larger, shrinking the airway.

Fig. 5 : Évaluation des voies aériennes supérieures chez deux patients.

a : voie aérienne étroite en raison surtout de l'ossature maxillomandibulaire.

b : tissus mous plus importants, avec réduction des voies aériennes. 
posterior facial compression is seen where both maxilla and mandible are retruded. However, when compared with the patient on the right side, who has the same UA volume $\left(13 \mathrm{~cm}^{3}\right)$, the subject on the left has a smaller transverse UA section, half the size $\left(0.59 \mathrm{vs} 1.29 \mathrm{~cm}^{2}\right)$. In the literature, there is evidence that a restriction of UA is the cause of OSA [22]. Although there was no UA caliber decrease in the subject on the right, a marked increase of the soft tissues volume was observed. This observation directs the diagnosis towards the soft palate (sagittal section of $5.36 \mathrm{~cm}^{2}$ ) causing a UA restriction and suggests treatment by ENT surgery. As for the patient on the left, bimaxillary protrusion surgery to treat OSA should be recommended. For the orthodontist, the salient parameters to be observed on the CBCT are: the bone volumes (maxilla and mandible), the sagittal section of the soft palate and the smallest transverse UA section.

\section{Conclusion}

While evaluating craniofacial bony structures and soft tissues in patients with suspected OSA disorder, CBCT can provide a fast, inexpensive and straightforward three-dimensional analysis of the upper airway. The role of craniofacial bone development in OSA is largely defined by the maxillo-palatine core volume, which is smaller in OSA patients after age, gender, height, neck circumference and BMI adjustment. Modern management should consider craniofacial anatomy in addition to clinical and endoscopic evaluations, hence actually reflecting the complexity of multi-level obstruction.

\section{Disclosure of interest}

The authors declare that they have no competing interest. petite des VAS. Chez le patient à gauche, une compression faciale postérieure est visible à l'endroit de la rétrusion du maxillaire et de la mandibule. Cependant, comparée au patient de droite, qui a le même volume des VAS $\left(13 \mathrm{~cm}^{3}\right)$, la section transversale la plus petite des VAS du sujet de gauche est de moitié inférieure $\left(0,59\right.$ vs $\left.1,29 \mathrm{~cm}^{2}\right)$. Dans la littérature, il n'existe pas de preuves confirmant qu'une restriction des VAS soit à l'origine de l'AOS [22]. Malgré l'absence de diminution de calibre des VAS chez le sujet de droite, une augmentation prononcée du volume des tissus mous a été observée. Ce constat oriente le diagnostic vers le palais mou (surface de section sagittale de $5,36 \mathrm{~cm}^{2}$ ) provoquant un rétrécissement des VAS, ce qui semblerait indiquer une chirurgie ORL. Pour l'orthodontiste, les paramètres les plus importants à observer sur le CBCT sont : les volumes osseux (maxillaire et mandibule), la section sagittale du palais mou et la section transversale la plus restreinte des VAS.

\section{Conclusion}

Lors d'évaluations des structures osseuses craniofaciales et des tissus mous chez les patients soupçonnés d'être atteints d'une AOS, le CBCT peut fournir une analyse tridimensionnelle rapide, simple et peu onéreuse des voies aériennes supérieures. Le rôle du développement des os craniofaciaux en AOS est en grande partie défini par le volume de base maxillopalatin, qui est plus réduit chez les patients avec AOS après ajustement pour l'âge, le sexe, la taille, la circonférence du cou, et l'IMC. La prise en charge moderne de l'apnée obstructive du sommeil devrait tenir compte de l'anatomie craniofaciale aussi bien que des évaluations cliniques et endoscopiques, et répondre ainsi à la complexité d'une obstruction à multiples facettes.

\section{Déclaration de liens d'intérêts}

Les auteurs déclarent ne pas avoir de liens d'intérêts.

\section{References/Références}

1. Peppard PE, Young T, Barnet JH, Palta M, Hagen EW, Hla KM. Increased prevalence of sleep disordered breathing in adults. Am J Epidemiol 2013;177:1006-14.

2. Kotecha BT, Hall AC. Role of surgery in adult obstructive sleep apnea. Sleep Med Rev 2014;18:405-13.

3. Cohen-Levy J, Petelle B, Vieille E, Dumitrache M, Fleury B. Changes in facial profile after maxillomandibular advancement surgery for obstructive sleep apnea syndrome. Int Orthod 2013;11:71-92. 
4. Strollo Jr. P, Soose RJ, Maurer JT, et al. Upper-airway stimulation for obstructive sleep apnea. N Engl J Med 2014;370:139-49.

5. Milano F, Billi MC, Marra F, Sorrenti G, Gracco A, Bonetti GA. Factors associated with the efficacy of mandibular advancing device treatment in adult OSA patients. Int Orthod 2013;11:278-89.

6. Kim JH, Guilleminault C. The nasomaxillary complex, the mandible, and sleep disordered breathing. Sleep Breath 2011;15:185-93.

7. Poirrier AL, Pire S, Raskin S, Limme M, Poirrier R. Contribution of postero-anterior cephalometry in obstructive sleep apnea. Laryngoscope 2012;122:2350-4.

8. Makdissi J. Cone beam CT in orthodontics: the current picture. Int Orthod 2013;11:1-20.

9. Chung RR, Lagravere MO, Flores-Mir C, Heo G, Carey JP, Major PW. A comparative analysis of angular cephalometric values between CBCT generated lateral cephalograms versus digitized conventional lateral cephalograms. Int Orthod 2009;7:308-21.

10. Ramírez-Sotelo LR, Almeida S, Ambrosano GM, Boscolo F. Validity and reproducibility of cephalometric measurements performed in full and hemifacial reconstructions derived from cone beam computed tomography. Angle Orthod 2012;82:827-32.

11. Strauss RA, Wang N. Cone beam computed tomography and obstructive sleep apnea. Aust Dent J 2012;57:61-71.

12. American Academy of Sleep Medicine. International classification of sleep disorders. 3rd ed. (ICSD-3); 2014 .

13. Pae EK, Lowe AA, Sasaki A, Price C, Tsuchiya A, Fleetham JA. A cephalometric and electromyographic study of upper airway structures in the upright and supine positions. Am J Orthod Dentofacial Orthop 1994;106:52-9.

14. Jankowski R. Revisiting human nose anatomy: phylogenic and ontogenic perspectives. Laryngoscope 2011;121:2461-7.

15. Guilleminault C, Lee JH, Chan A. Pediatric obstructive sleep apnea syndrome. Arch Pediatr Adolesc Med 2005;159:775-85.

16. Pirelli P, Saponara M, Guilleminault C. Rapid maxillary expansion (RME) for pediatric obstructive sleep apnea: a 12-year follow-up. Sleep Med 2015;16:933-5.

17. Villa MP, Castaldo R, Miano S, et al. Adenotonsillectomy and orthodontic therapy in pediatric obstructive sleep apnea. Sleep Breath 2014;18:533-9.

18. Van Holsbeke C, Vos W, Van Hoorenbeeck K, et al. Functional respiratory imaging as a tool to assess upper airway patency in children with obstructive sleep apnea. Sleep Med 2013;14:433-9.

19. Li HY, Wang PC, Chen YP, Lee LA, Fang TJ, Lin HC. Critical appraisal and meta-analysis of nasal surgery for obstructive sleep apnea. Am J Rhinol Allergy 2011;25:45-9.

20. Lowe AA, Gionhaku N, Takeuchi K, Fleetham JA, Three-dimensional CT. reconstructions of tongue and airway in adult subjects with obstructive sleep apnea. Am J Orthod Dentofacial Orthop 1986;90:364-74.

21. Schwab RJ, Pasirstein M, Pierson R, et al. Identification of upper airway anatomic risk factors for obstructive sleep apnea with volumetric magnetic resonance imaging. Am J Respir Crit Care Med 2003;168:522-30.

22. Schendel SA, Hatcher D. Automated 3-dimensional airway analysis from cone-beam computed tomography data. J Oral Maxillofac Surg 2010;68:696-701. 